\title{
La testimonianza del minore: riflessioni criminologiche
}

\section{The minor witness: Criminological reflections}

\author{
Susanna Pietralunga* e Claudia Salvioli**
}

\begin{abstract}
Riassunto: La testimonianza del minore presenta aspetti di specifica complessità e la letteratura criminologica, psichiatrico-forense e giuridica analizza approfonditamente $i$ compiti dell'esperto sotto il profilo metodologico, clinico ed, altresì, deontologico. Si segnala per la sua complessità la particolare situazione che si crea allorché il minore, non di rado all'interno di un reato di gruppo, riveste il duplice ruolo di testimone e di autore di reato. Anche gli importanti cambiamenti che interessano la cultura sociale e le sollecitazioni che derivano dalla comunicazione multimediale possono facilitare ulteriormente l'intrecciarsi dei ruoli di testimone ed autore di reato. Si pongono in tali contesti problemi specifici ai quali la riflessione criminologica può fornire un contributo, segnatamente nella prospettiva della prevenzione primaria e secondaria.
\end{abstract}

Parole Chiave: testimonianza del minore, sviluppo del minore, tutela del minore, transizione culturale, relazione genitore-figlio.

Abstract: Minor's testimony is a complex issue, and the criminological, psychiatric-forensic and legal literature analyzes the role of the expert from a methodological, clinical and, also, deontological point of view. The minor, not infrequently within peers, has the dual role of witness and author of the crime. Roles of witness and author of crime can coincide with important changes in social culture and the effects of multimedia communication: Criminology can make a contribution to these specific problems through primary and secondary prevention.

Key Words: minor witness, minor's development, minor's protection, cultural transition, family relationships.

\footnotetext{
* Professore Associato di Criminologia, Dipartimento di Educazione e Scienze Umane, Università degli Studi di Modena e Reggio Emilia.

** Specialista in Criminologia Clinica, Educatore Coordinatore, Centro di Giustizia Minorile della Regione Emilia Romagna (Bologna).
}

Ricevuto 01. 04.2021 Accettato 14.04.2021 Submitted 01.04.2021 Accepted 15.04.2021

RSF (ISSN 1129-6437, ISSNe 1972-5582), VOL. CXLV, 2021, 1, Doi: 10.3280/RSF2021-001004 Copyright (C) FrancoAngeli

N.B: Copia ad uso personale. È vietata la riproduzione (totale o parziale) dell'opera con qualsiasi mezzo effettuata e la sua messa a disposizione di terzi, sia in forma gratuita sia a pagamento. 


\section{La testimonianza del minore: la rilevanza e la complessità del tema nella letteratura criminologica, psichiatrico-forense e giuridica}

Il tema della testimonianza del minore riveste una rilevanza estrema per la sua evidente delicatezza e complessità, dal momento che attiene ad un soggetto che occupa, all'interno del gruppo sociale, una posizione all'un tempo di particolare fragilità e di particolare valore, poiché i giovani di oggi costituiscono il gruppo sociale del domani. Il tema è perciò largamente trattato nella letteratura scientifica sia in ambito psichiatrico-forense che criminologico e giuridico, nelle molteplici dimensioni di analisi della valutazione clinica dell'idoneità mentale a rendere testimonianza, della metodologia dell'intervista al minore e dell'intervento peritale, con particolare riguardo al momento della descrizione dell'abuso, cui si sommano le riflessioni sugli aspetti deontologici di tali complessi interventi.

Sotto il profilo delle tesi proposte dalla letteratura scientifica in materia di testimonianza del minore si è passati, nel tempo, da una posizione consolidata e prevalente in un passato tutt'altro che remoto e che arriva - sostanzialmente - fino alla metà del secolo scorso, fondata su una complessiva percezione di sostanziale scarsa attendibilità della testimonianza del minore, ad una posizione orientata a ritenere che la testimonianza di un bambino anche molto piccolo sia del tutto attendibile, fino ad una posizione di più recente maggiore attenzione, rivolta ad analizzarne approfonditamente sia i contenuti che le condizioni nelle quali essa viene raccolta, con specifico riguardo ai fattori di influenzamento ed ai metodi per l'ascolto dei minori [1,2].

Si evidenzia in particolare sotto il primo profilo, l'importanza da parte del perito di contestualizzare i fatti che hanno determinato l'intervento dell'esperto, ma anche di ricostruire le notizie relative al fatto di reato attraverso una attenta disamina degli atti processuali definendo gli accadimenti, analizzando sia le fonti dirette che indirette di informazione, raccogliendo dati sulle caratteristiche ambientali della presunta vittima, senza mancare di valutare le possibili fonti di errore [3].

In tema di idoneità mentale a rendere testimonianza, le sintesi messe a fuoco dalla letteratura scientifica [4] evidenziano come la valutazione debba articolarsi ed estendersi sia alla disamina delle funzioni intrapsichiche del soggetto sia, ed altresì, all'approfondimento delle caratteristiche del suo ambiente sociale, familiare, relazionale e culturale, anche grazie ai dati delle ricerche svolte su tali temi nell'ambito dell'età evolutiva [5].

Fra le condizioni più significative e rilevanti, inoltre, per l'attendibilità della testimonianza del minore è stato posto in evidenza il ruolo che egli ha svolto nella vicenda e quanto l'avervi partecipato come protagonista incida positivamente sul ricordo [3]. 
Nello stesso ambito è stata, altresì, sottolineata la rilevanza rivestita dal trascorrere del tempo: qualora il momento dell'evento sia particolarmente lontano, i ricordi e le risposte diminuiscono ed aumentano gli errori [6].

Si segnalano, altresì, situazioni complesse nelle quali il minore riveste il duplice ruolo di vittima e di testimone, frequenti in particolare all'interno dei reati verso i minori in ambito familiare caratterizzati, com'è noto, da un numero oscuro particolarmente elevato e, perciò, dall'assenza di testimoni. Particolare importanza, a questo riguardo, è stata attribuita alla necessità di evitare attentamente l'uso di domande suggestive, così come il ricorso a domande direttive privilegiando invece modalità di racconto libere e spontanee da parte del bambino, soprattutto nei bambini in età prescolare [7, 8, 3].

In tema di metodologia dell'intervista si sottolinea, ancora, la necessità di prestare particolare attenzione all'eventuale presenza di fraintendimenti, di esagerazioni, di menzogne, del desiderio di proteggere l'abusante o di dichiarazioni c.d. "a reticolo", allorché le denunce si sovrappongono parzialmente in casi in cui sono presenti più presunte vittime e più presunti abusanti [3].

Si è rilevata la necessità dell'organizzazione delle domande [9], e come la maggior quantità di risposte si ottenga attraverso le domande dirette [10].

A proposito della specifica tutela del minore testimone in ambito procedimentale, non si può non ricordare la "Carta di Noto", documento redatto nel 1996 e modificato nel 2002 [11] ed ancora nel 2007 (Protocollo di Venezia), che raccoglie le linee guida per l'indagine e l'esame psicologico del minore vittima di reato a sfondo sessuale. L'atto, nato dalla collaborazione interdisciplinare di magistrati, avvocati, psicologi, psichiatri, neuropsichiatri infantili, criminologi e medici legali, è volto a fornire linee guida al modus operandi in caso di ascolto del minore, anche se con specifico riferimento a testimoni minorenni vittime di abusi sessuali [12].

Pur - e certamente - al di fuori di ogni rigidità applicativa, si deve sottolineare l'utilità che essa può rivestire come punto di riferimento in questo ambito valutativo di particolare delicatezza e complessità, sia per gli esperti che sono chiamati a compiere tali indagini ma anche per gli appartenenti al mondo giudiziario (magistrati e difensori), e vanno segnalate le rilevanti applicazioni ed indicazioni che ne sono state fatte in sede giurisprudenziale [3].

\section{La testimonianza del minore autore di reato}

Vanno sottolineate per la loro specificità, e rivestono particolare interesse e delicatezza, le situazioni nelle quali il minore ricopre il duplice ruolo di 
testimone e di autore di reato. Si tratta, d'altro canto, di circostanze tutt'altro che infrequenti, proprio per la condizione del tutto specifica dell'età minorile, con l'influenzabilità che la connota anche rispetto agli adulti e con la rilevanza centrale rivestita dal gruppo dei pari.

Il criterio che pervade il procedimento penale minorile è ispirato, in primis, al secondo comma dell'art. 31 della Costituzione, in base al quale la Repubblica "Protegge...l'infanzia e la gioventù favorendo gli istituti necessari a tale scopo". Tale tutela trova peraltro le sue radici anche in documenti internazionali, quali le "Regole minime delle Nazioni Unite per l'Amministrazione della Giustizia Minorile”, la "Raccomandazione n. 20 del Comitato dei Ministri del Consiglio d'Europa circa le reazioni sociali della delinquenza minorile”, approvata nel 1987, la Convenzione Onu sui diritti dell'infanzia del 20 novembre 1989. Proprio in quest'ultima viene specificamente enunciato il diritto del minore alle garanzie processuali [12].

Nel rispetto di tale approccio ispirato alla tutela, quando il minore che deve essere ascoltato è autore di reato la sua testimonianza deve necessariamente e preliminarmente coordinarsi con il diritto alla difesa, poiché a renderla è un soggetto fragile, un adolescente, che per di più riveste un doppio ruolo, ugualmente complicato da affrontare in entrambe le sue posizioni e prospettive. Si tratta pertanto di situazioni che presentano particolare complessità e che racchiudono molte variabili intrecciate tra loro e non di rado tra loro confliggenti. Per quanto concerne la garanzia del diritto di difesa, la Raccomandazione del Consiglio d'Europa n. 20 del 1987 [13] esorta i governi a rivedere, se necessario, le proprie legislazioni inerenti i procedimenti contro i minori rafforzando le garanzie legali durante i procedimenti, compresa la fase delle indagini di polizia.

La Costituzione italiana garantisce appieno ed in senso ampio i suddetti principi di tutela del minore nelle previsioni in cui - oltre ad enunciarsi i diritti inviolabili dei quali egli, in quanto persona, è titolare - si delinea una concezione di famiglia come formazione sociale finalizzata alla promozione e allo sviluppo dei suoi componenti.

Il nostro Paese ha recepito appieno le indicazioni degli Atti internazionali relativi alla tutela del minore anche in ambito penale, con la promulgazione del D.P.R. 448/88 sul processo penale minorile e la previsione di alcune disposizioni contenute nel Codice di Procedura Penale [13].

Il D.P.R. 448/88 sul processo penale minorile, corpus iuris ad hoc, è fondato sul principio generale di centralità del minore, impostazione che si evince da una serie di istituti orientati al sostegno delle fragilità ed alla implementazione delle risorse, in una visione che vede la stessa funzione punitiva come strumento pedagogico. Tali principi cardine trovano espressione applicativa in un impianto di politica penale che si traduce in coerenti indirizzi operativi, 
quali il ricorso alla carcerazione come ultima ratio e quindi la minor lesività della pena, la rapida fuoriuscita dal circuito processuale, la non interruzione dei percorsi educativi in atto, l'attiva partecipazione del minore nelle diverse fasi processuali per tutelare il suo diritto alla comprensione - in una chiave di responsabilizzazione attraverso la lettura e l'interpretazione della genesi del proprio agire - l'individualizzazione del trattamento, per rispondere a quegli specifici bisogni di quel dato soggetto al fine di facilitarne la crescita armoniosa. Ciò, risulta particolarmente evidente nell'istituto della sospensione del processo con messa alla prova, concepito come un percorso di impegno più che di risultato, a differenza dell'analoga previsione legislativa relativa agli adulti, ai quali viene richiesto un concreto e tangibile prodotto della propria volontà di reinserimento [14-17].

Istituto di particolare significatività appare la mediazione penale, che tende a mostrare 'l'Altro da sé' ai protagonisti dell'evento reato, in una circolarità che diviene uno strumento contro l'autocentrismo, il narcisismo e la volontà di soddisfare le proprie istanze anche a scapito dell'altro, spinte pulsionali all'azione che caratterizzano l'adolescenza. Nella mediazione l'obiettivo è quello di traslare dall'agito violento al riconoscimento dell'altro e della sua sofferenza attraverso la decodifica del 'sentire'. Tale istituto appare particolarmente efficace nei reati di maltrattamento intra-familiare ove il minore è autore, fattispecie che sta conoscendo una particolare e preoccupante ingravescenza. In tale ottica, il percorso di mediazione penale produce una valorizzazione del ruolo della vittima, evidenziando in modo speculare il carattere sistemico e relazionale del contenitore-famiglia e dell'impianto dell'istituto [18, 19, 20, 21, 22, 23].

La tendenza attuale in molti Paesi, in risposta ad una forte richiesta di sicurezza sociale, è quella di trascurare misure educative in favore di risposte punitive e non inclusive che non offrono tutela sia in termini di general che di specialprevenzione. Molti Paesi valutano o hanno già attuato un abbassamento dell'età imputabile, nella direzione di una confusività tra semplici abilità, oggi in effetti precocemente acquisite dal minore, e vere e proprie competenze psico-sociali-affettive, contenuti ben più complessi, riassunti nel concetto di 'maturità' che ha ispirato il nostro legislatore [1S].

Il nostro ordinamento, proprio nell'ottica di considerare il minore un soggetto competente, ma non ancora adulto, assicura al ragazzo in ogni stato e grado del procedimento, oltre che la presenza dei genitori, l'assistenza dei servizi minorili del Ministero della Giustizia e di quelli istituiti presso gli enti locali (Comune, ASL, Servizi per le Dipendenze, Neuropsichiatria Infantile), allo scopo di svolgere 'l'inchiesta sulla sua personalità' per poi formulare un idoneo percorso trattamentale [24].

Tale principio cardine trova piena espressione nell'art. 9 del detto DPR, 
il quale, al primo comma, dispone che: "Il pubblico ministero e il giudice acquisiscono elementi circa le condizioni e le risorse personali, familiari, sociali e ambientali del minorenne al fine di accertarne l'imputabilità e il grado di responsabilità".

È evidente come tra la giustizia minorile e quella ordinaria esista una vera e propria inversione del presupposto di base: la conoscenza della persona è l'assunto cardine per il giudice minorile che gli consente di calibrare sulle caratteristiche e sui bisogni di ciascun ragazzo un percorso di ricostruzione, traendo spunto dalla specifica situazione di partenza. Al contrario, il giudizio su un adulto è relativo solo a ciò che è stato. Anche il disposto dall'art. 133 c.p. in merito alla discrezionalità del giudice, fa riferimento ad indicatori comunque evidenziatisi nel quadro dei trascorsi. La perizia sulla personalità dell'imputato è espressamente proibita, pertanto i percorsi di crescita personale potranno essere ipotizzati e costruiti solo in fase esecutiva. L'indagine sulla personalità del ragazzo, oltre che per l'accertamento della sussistenza della capacità di intendere e volere in capo all'imputato minorenne, è quindi indispensabile per individuare la risposta trattamentale più adeguata ad ottenere i risultati educativi più efficaci $[25,26]$.

Alla luce delle suddette tutele - che assumono la fragilità e la necessità di un' adeguata e sempre attualizzata lettura individuale e sociale dell'adolescenza - risulta evidente come il tema sul quale si intende stimolare una riflessione, la testimonianza del minore imputabile autore di reato, presenti particolare complessità, peculiarità specifiche, aspetti di delicatezza e difficoltà differenti da quelli connessi all'ascolto dell'infraquattordicenne o alla testimonianza dell'adulto.

Da un lato, il legislatore ha inteso dare rilevanza a ciò che il minore può riferire e, al tempo stesso, lo ha valorizzato e responsabilizzato. Ciò in inversione di tendenza rispetto al passato, quando l'attenzione verso il minorenne nel ruolo di testimone - ma anche di parte lesa - risultava residuale, come era dato evincere nel Codice di procedura penale del 1930, ove l'unica norma che accennava ai minori era l'art. 449, la quale escludeva l'obbligo di prestare giuramento per i minori di anni 14. L'obbligo di celebrare il giudizio a porte chiuse non era previsto neppure per i reati a sfondo sessuale: la scelta era affidata alla discrezionalità del giudice per ragioni di sicurezza, di ordine pubblico o moralità.

Dal punto di vista meramente procedurale, occorre ben distinguere le situazioni in cui il minorenne è autore, vittima o testimone oculare di un reato, benché - come si dirà - talune situazioni non siano così nettamente categorizzabili e presentino invece sfumature che rendono in parte sovrapponibili le posizioni del ragazzo stesso rispetto al fatto [27, 28, 2S].

In merito al modus del momento testimoniale di un minore, è stato 
sottolineato in dottrina come il termine "ascolto" - utilizzato di frequente anche dagli addetti ai lavori, in luogo di quello tecnicamente più corretto di "testimonianza" - risulti di particolare efficacia poiché rimanda ad un'azione che va oltre il semplice 'udire', per allargarsi in uno spettro che riveste, appunto, l'accezione di 'accoglienza del dire' [29].

Se è di tutta evidenza come il bambino richieda questo tipo di approccio - dovendosi usare particolari cautele e risultando talora necessario decrittare il suo stesso linguaggio - anche l'adolescente richiede diverse, ma non meno indispensabili, attenzioni.

Nei casi in cui il minore sia semplicemente testimone oculare di un reato, l'art. 196 c.p.p. recita che "i minori (compresi coloro che abbiano meno di quattordici anni) hanno la capacità di testimoniare". Il contenuto della disposizione viene parzialmente mitigato da quella che prevede l'obbligo di impegnarsi a dire la verità nel rendere una testimonianza nel processo per le persone che hanno compiuto quattordici anni [27, 30-34].

Per un minore l'ascolto in seno ad un procedimento penale rappresenta un momento foriero di molte possibili tensioni e paure. Per questo è di estrema importanza comprendere appieno e correttamente ciò che il minore dichiara $\mathrm{e}$ inquadrarlo nel contesto soggettivo del ragazzo stesso, pur nelle irrinunciabili esigenze di accertamento della verità [35, 36, 27, 3S].

Sono molte le variabili che possono influenzare la testimonianza di un adolescente.

Si è sottolineato, infatti, come frequentemente le situazioni nelle quali il minore assiste al fatto sul quale riferisce lo vedono partecipe con un ruolo più o meno attivo, rivestendo una parte che può qualificarlo come correo, come testimone, come persona informata sui fatti [27, 28].

Tali differenti posizioni si originano con particolare frequenza nei reati commessi in gruppo, modalità che le statistiche evidenziano come maggiormente ricorrente per quanto attiene alcune fattispecie, come il danneggiamento, i reati di tipo appropriativo (rapine, furti, estorsioni), i delitti legati alla cessione di sostanze stupefacenti - soprattutto per i minori che ne sono anche consumatori - ma anche reati contro la persona, come violenze sessuali, violenze private, risse.

$\mathrm{Vi} \mathrm{sono} \mathrm{situazioni} \mathrm{illecite} \mathrm{alle} \mathrm{quali} \mathrm{il} \mathrm{giovane} \mathrm{assiste} \mathrm{e} \mathrm{nelle} \mathrm{quali} \mathrm{si} \mathrm{trova}$ coinvolto penalmente pur non esercitando un ruolo attivo: perché non ha preso le necessarie precauzioni o le dovute distanze - rientrando così nella fattispecie ex art. 40, comma 2 c.p., in forza del quale "non impedire l'evento che si ha l'obbligo giuridico di evitare equivale a cagionarlo" - o perché non ha prestato il dovuto soccorso alla vittima, violando l'art. 593 c.p., relativo all'omissione di soccorso.

All'interno di questo scenario complesso di possibili ruoli formali e 
sostanziali che il ragazzo può rivestire, esaminiamo in quali circostanze il minore autore di reato si possa trovare ad assumere le vesti del testimone.

Dall'incipit del suo percorso processuale penale - in occasione dell'udienza di convalida se arrestato, oppure di interrogatorio di garanzia se sottoposto a misura cautelare dalla libertà o denunciato a piede libero - al minore indagato vengono chiarite le sue responsabilità attraverso i dovuti avvisi di legge, come previsto dall'art. 64 c.p.p. In riferimento a sé, il ragazzo è libero di riferire ciò che ritiene - come anche di avvalersi della facoltà di non rispondere senza incorrere in nessuna conseguenza, essendo tenuto, in questi casi, a dire la verità solo relativamente alle proprie generalità, secondo il brocardo nemo tenetur se detegere.

Qualora il ragazzo riferisca rispetto a fatti che coinvolgono altre persone, invece, egli assume il ruolo di testimone ed in questa veste può incorrere nella commissione del reato di falsa testimonianza, se non risulti veritiero ciò che riporta (art. 372 c.p.).

La posizione di testimone deve essere tuttavia valutata tenendo conto dei casi di incompatibilità ex artt. 12 e 197 c.p.p., in merito alla connessione del reato: non è possibile testimoniare rispetto a qualcuno, se il reato di cui si risponde è contiguo a quello della persona oggetto di testimonianza, cioè se vi sia tra i due una stretta connessione, come ad esempio nel caso di coimputati in un reato commesso in concorso.

Tale incompatibilità, tuttavia, non sussiste nei casi in cui si tratti di connessione teleologica, che si determina quando il reato per cui si procede viene commesso per eseguirne od occultarne altri. In questa eventualità, la testimonianza del minore è ammessa, tuttavia verrà raccolta in modo 'garantito', con l'assistenza del difensore.

L'incompatibilità della testimonianza tra coimputati viene meno anche qualora la posizione processuale del minore testimone correo sia definitiva (art. 197 bis c.p.p.), sul presupposto che in questo caso il ragazzo non abbia più alcun interesse ad orientare i fatti a proprio vantaggio o comunque in direzioni non aderenti alla verità. Il trovarsi in posizioni o fasi processuali diverse è circostanza che si verifica non di rado tra correi minorenni, poiché i percorsi possono frequentemente differenziarsi per esiti, istituti applicati e durata, nella piena realizzazione della individualizzazione trattamentale [37, 38].

Come già sottolineato, queste particolari situazioni si producono spesso nei reati di gruppo, essendo frequentemente sfumato o sovrapposto il ruolo che ciascuno assume rispetto all'altro ed essendo differenti le progettualità trattamentali, che verranno proposte a ciascuno.

Come testimone, il minore verrà valutato per accertarne la credibilità, considerando la maturità, la personalità, il contesto familiare, i vissuti che gli 
appartengono, nonché il suo rapporto con la persona rispetto alla quale egli rende testimonianza.

Quest'ultimo aspetto riveste una particolare importanza, poiché nelle dinamiche tra adolescenti sono spesso presenti influenzamenti, convincimenti, dinamiche di prevaricazionelsubordinazione 0 , al contrario, di solidarietà. Potrebbe occorrere decodificare nel minore testimone la ferrea convinzione di non dover commettere una 'infamia', un tradimento nei confronti di un amico, in una mal interpretazione del concetto di lealtà verso un proprio pari, a detrimento di quella verso la verità $[39,40]$. Parimenti, la testimonianza potrebbe essere condizionata e inficiata dal rapporto esistente tra il ragazzo e un appartenente al proprio gruppo, qualora il primo si trovi in una posizione di subalternità rispetto al secondo. Ciò potrebbe causare nel teste timore e disagio, con conseguente vizio nell'esposizione dei fatti: si pensi alla testimonianza nei confronti di una figura vissuta come leader, sostenuta da tutto il gruppo, che il minore si troverebbe poi a fronteggiare, da solo; oppure la deposizione relativa ad un adulto, un soggetto con una posizione delinquenziale consistente, che ha un aggancio nella vita del minore, come ad esempio il fornitore di stupefacenti, poi consumati o ceduti dal minore stesso.

La valutazione dell'attendibilità del testimone deve essere effettuata dal giudice, con prevalenza sulle perizie formulate da esperti chiamati a valutare la capacità del minore a deporre (art. 196 c.p.p.).

Si è visto come il minore possa testimoniare rispetto ad altro soggetto solo nelle suddette ipotesi di connessione teleologica o di definitività della sua posizione processuale. Nella chiamata in correità, quando trattasi di minore, sarà particolarmente accurato il vaglio di alcune variabili, da valutare insieme alle caratteristiche individuali e sociali sopra menzionate, quali la precisione, la coerenza, la spontaneità della testimonianza. Tutti i fattori andranno poi supportati con altre risultanze conoscitive tali da confermarne l'attendibilità $[31,36]$.

La posizione del minore in queste circostanze è di evidente delicatezza e complessità. Da un lato il legislatore ha inteso valorizzare ciò che il minore può riferire, altresì tale riconoscimento comporta imprescindibili cautele nella valutazione del soggetto, il quale porta con sé, inevitabilmente, una serie di variabili che vanno ad intrecciarsi, con ricaduta sulla sua capacità di intendere e di volere, in primis, e sulla sua attendibilità e libertà testimoniale in secundis.

Una situazione di particolare complessità e difficoltà si viene a creare quando il minore sia chiamato a testimoniare in virtù della sua appartenenza alla sottocultura della criminalità organizzata. Sappiamo come tale contesto, caratterizzato da una struttura delinquenziale rigidamente gerarchizzata e da un senso di appartenenza altamente uncinante e collusivo, veicoli regole e 
modelli non conformi alla società, inducendo facilmente un’assimilazione da parte del minore di principi etici “altri”; l'agito delittuoso deriva da modelli introiettati di tipo antisociale, che in una lettura psicodinamica comporteranno la costruzione di un Super-io criminoso [41].

Chiedere all'adolescente di discostarsi dall'assetto culturale di derivazione, di contrapporsi alle figure genitoriali e al gruppo sociale di appartenenza, di effettuare persino una revisione critica dell'impianto culturale che caratterizza le proprie origini, 'tradendole', significa esigere una risposta - il più delle volte prematura - in grado di generare una frattura interna: al minore si prospetteranno un senso di solitudine e di fragilità ed uno smarrimento molto spesso indecifrabili ed intollerabili. Una scelta simile, già difficoltosa per un adulto, per un minore può costituire l'origine di uno strappo interiore insanabile, stante l'ancora incompleta strutturazione di personalità e l'intreccio tra lealtà verso i legami familiari e lealtà verso la Giustizia.

Nell'ottica di tutelare i minori provenienti da famiglie che appartengono alla criminalità organizzata, e quindi - inscindibilmente - nell'intento di preservare e curare i legami 'recuperabili', per non recidere tutti i vincoli affettivi dei giovani che altrimenti si sentirebbero privi di radici, sono sorti percorsi istituzionali di supporto e di sostegno, con l'obiettivo di accompagnare il ragazzo ed i suoi familiari estranei alla commissione di crimini verso una presa di distanza e quindi verso la rinascita. In questa direzione opera l'Associazione "Libera", la quale, oltre ad occuparsi di coordinamento della progettazione e della relativa concretizzazione in singoli percorsi familiari, cura l'individuazione di una proficua destinazione dei beni confiscati alle mafie. I progetti che sono destinati a minori appartenenti a clan mafiosi, prevedono la tutela anche di membri del nucleo familiare estranei al contesto mafioso o con la volontà di discostarsene. Si tratta spesso di madri e fratelli dei ragazzi, considerati bisognosi di sostegno per ricostruire la propria esistenza lontano da un sistema altamente pericoloso, dal quale, autonomamente, sarebbe difficilissimo affrancarsi.

Un altro caso di testimonianza che presenta specifiche complessità è quello dei minorenni stranieri, per i quali si pongono delle particolari difficoltà a partire dai più immediati problemi relativi alla comprensione linguistica, fino agli aspetti più complessi legati all'identità culturale, fattore foriero di un possibile conflitto o comunque di una differente interpretazione della stessa realtà fattuale $[42,43]$. In queste circostanze il contributo del mediatore culturale assume particolare rilevanza. Nei reati di violenza sessuale, ad esempio, lo scontro con appartenenze culturali, che danno una lettura del delitto molto lontana dai principi di tutela della persona e della donna alla base della nostra cultura giuridica e socio-culturale, determina una difficoltà 
nella decodifica dell'accaduto. Nel caso lo straniero appartenente a questo tipo di culture sia un testimone o l'autore, occorrerà decifrare e mostrare il rifiuto opposto dalla vittima per ciò che è, cioè un 'no', presupposto stesso della violenza e quindi dell'esistenza del reato; nel caso sia la vittima, occorrerà sostenerla nel percorso di presa di coscienza di essere tale, sentendosi tale.

Accade di frequente che minorenni stranieri chiamati a testimoniare, denunciati o arrestati siano sprovvisti di documenti oppure siano in possesso di documenti non validi. La corretta attribuzione dell'età è fondamentale per garantire le dovute tutele al minore: l'art. 8 del D.P.R. 448/88 recita: “Quando vi è incertezza sulla minore età dell'imputato, il giudice dispone, anche d'ufficio, la perizia. Qualora, anche dopo la perizia, permangano dubbi sulla minore età, questa è presunta ad ogni effetto", secondo il principio in dubio pro reo. Per dirimere i dubbi in merito all'età sono indispensabili valutazioni mediche, quali misurazioni antropometriche, radiografie - in genere polso e gomito - rilievi dentari. È però importante sottolineare come siano state riscontrate difficoltà nella valutazione di tali accertamenti, stante la plausibile differente gradazione di sviluppo in quei giovani che presentino trascorsi di malnutrizione o altre privazioni incidenti sulla crescita. Sempre in ottica garantista è da leggersi l'obbligo previsto dal nostro ordinamento di informare la rappresentanza diplomatica o consolare del paese cui appartiene il minore straniero, quando venga adottato nei suoi confronti un provvedimento in materia di libertà personale o di tutela, tranne nel caso in cui questi abbia fatto richiesta di asilo [44, 45].

$\mathrm{Si}$ è sottolineato come i reati di gruppo rappresentino un'alta percentuale di quelli commessi da minorenni e come questo fattore ponga questioni rilevanti sotto il profilo procedurale e sostanziale della testimonianza del minore.

Numerosi studi, a partire dalla prima metà del ' 900 fino ai giorni nostri, hanno indagato quali siano i fattori favorenti questo fenomeno, trasversale a differenti contesti storico-culturali. Molti dei primi studi sociologici hanno interpretato la banda delinquenziale come un fenomeno sottoculturale, e quindi come espressione di norme e valori tipici di gruppi sociali appartenenti a classi deprivate, così come illustrato dai più autorevoli studiosi della prima metà del 1900, quali Thrasher, Cohen, Cloward e Ohlin. La lettura tradizionale legata a questi studi vede il gruppo connotato in senso deviante, in genere costituito da soggetti appartenenti a contesti socio-familiari degradati o disgregati, comunque carenti nelle loro funzioni educative. Tale condizione rende l'adolescente portatore di una particolare solitudine affettivo-emotiva e il disperato bisogno di appartenenza che ne deriva trova facile risposta nel gruppo, contenitore protettivo e rassicurante, che offre senso di condivisione, legame, accoglimento e accettazione. Sentirsi 'uguale agli altri' e dagli altri 
'visto' e 'riconosciuto', significa trovare un'identità. La dimensione d'insieme può diventare un luogo dove è più facile stare, rispetto alla famiglia, alla scuola, alla società [14]. L'appartenenza deve essere sancita e identificabile, così da opporla agli 'altri': i membri del gruppo osservano il conformismo alle regole del gruppo stesso, spesso utilizzano un linguaggio con specifici codici comunicativi e fanno ricorso ad elementi estetici che li rendono immediatamente riconoscibili e riconducibili all'insieme, in un utilizzo della forma che diviene sostanza.

La vittima del reato di gruppo è spesso un altro soggetto minorenne o comunque un soggetto debole. Nei reati di tipo appropriativo e di danneggiamento, l'agito risulta essere di frequente l'espressione di una rabbia sottesa, di una volontà di riscatto, di rivolta all'umiliazione derivante dalla non integrazione. A muovere il gruppo non è tanto la volontà di impossessarsi dell'oggetto o di danneggiarlo, quanto quella di ottenere o distruggere ciò che l'oggetto rappresenta. L'oggetto sottratto o danneggiato, diviene il catalizzatore della frustrazione per ciò che non si è, prima ancora che per ciò che non si ha: la casa, la famiglia, il riconoscimento sociale, l'apprezzamento. Le condotte devianti, le modalità oppositive e provocatorie mascherano così la paura di non essere adeguati, di non essere amati, né - soprattutto - degni di esserlo [4S].

Attualmente, tuttavia, il fenomeno del gruppo delinquente risulta essere più complicato e sfaccettato, in simmetria con un contesto storico, sociale, culturale ed economico estremamente complesso. È indubbio che, indipendentemente dall'ambiente socio-culturale di appartenenza, l'adolescente attraversi un momento di crescita che presenta peculiari fragilità. L'adolescenza è infatti una fase dello sviluppo delicata, complessa ed articolata, caratterizzata dal succedersi di continui cambiamenti evolutivi attraverso i quali l'individuo cerca di consolidare e definire la propria identità, chiedendo l'allentamento della sfera di controllo degli adulti ed esperendosi nel mondo esterno, per poi giungere alla maturazione attraverso l'emancipazione e l'affrancamento dalle figure genitoriali. Il ragazzo - in modo più o meno marcato e oppositivo tende a mettere in discussione l'impianto normativo familiare e, non di rado, anche quello sociale. In questo momento di crescita, la spinta a riunirsi in gruppo è sostenuta dalla ricerca della propria identità per mezzo del confronto e del rispecchiamento nell'altro. Il gruppo adolescenziale offre al giovane nuove regole e nuovi valori da contrapporre a quelli introiettati e le 'leggi' del gruppo, condivise dal gruppo, hanno una valenza e una significatività tali da divenire vincolanti per i singoli membri e da orientare i loro atteggiamenti e comportamenti, anche in direzione antisociale [46].

Tale aspetto riveste particolare importanza nell' analisi criminogenetica della delinquenza giovanile, poiché incide sul riconoscimento della responsabilità 
individuale, che all'interno del gruppo diviene 'diffusa', distribuita tra tutti e come tale meno pesante e percettibile, caratterizzata talora da una vera e propria dispercezione in minus.

In particolare, i reati che si connotano per discontrollo o violenza possono facilmente essere favoriti dal contesto di gruppo, poiché l'insieme funge da alimentatore e al tempo stesso da dispersore di responsabilità.

È di tutta evidenza come il gruppo possa divenire un fattore diinfluenzamento soprattutto quando il singolo presenti un'identità ancora labile o altri elementi di fragilità, quali un ambiente familiare problematico, un iter scolastico accidentato, un percorso migratorio familiare o individuale alle spalle, la tossico o alcooldipendenza, la presenza di disturbi di tipo psicopatologico o altre variabili situazionali di tipo sociale o individuale.

Da tutto ciò consegue come la descritta pregnanza del gruppo sui membri si riverberi in modo considerevole anche sulla 'libertà' testimoniale che il singolo può avere o nei confronti di altri appartenenti al suo stesso gruppo o nei confronti dei membri di un gruppo 'contro' di lui.

Il fenomeno della delinquenza di gruppo, che sta vieppiù preoccupando l'opinione pubblica per la ingravescente ferocia e gratuità, oggi deve tuttavia essere accostato con una chiave di lettura che utilizzi un focus più ampio, non ponendo l'attenzione solo al vuoto valoriale del mondo giovanile, ma approfondendo le origini di tale vuoto. Il concetto di delinquens, cioè di colui che fallisce, manca al proprio dovere sociale, infrange le regole del gruppo attraverso la violenza, non sembra esaurirsi all'interno di contesti deprivati dal punto di vista sociale e culturale, appartenenti ad aree suburbane o geografiche problematiche. Tale ottica oggi risulta eccessivamente parcellizzante, apparendo più appropriata una visione delle cause sottese all'incontrollabilità dell'agire deviante giovanile, che faccia riferimento all'appartenenza a contesti socio-familiari non in grado di veicolare adeguati valori morali, non necessariamente per scarsità di mezzi economici o culturali. Il panorama storico-culturale contemporaneo appare permeato in modo trasversale da una grave crisi valoriale, che non sembra risparmiare nessuna fascia sociale, né essere appannaggio dell'età giovanile: la devianza adolescenziale appare, piuttosto, come l'espressione di una crisi sociale che riguarda in primis gli adulti e le istituzioni, che dagli adulti sono composte. Negli ultimi anni, in effetti, assistiamo a fenomeni delinquenziali giovanili, che interessano sempre più anche classi sociali medio-alte: i reati stessi riflettono tale tendenza, come dimostrano le frequenti risse tra adolescenti benestanti ed annoiati in prestigiosi locali, le violenze sessuali di gruppo, le azioni violente nei confronti di soggetti deboli - come disabili, senzatetto, minoranze vessate per le proprie origini o l'orientamento sessuale - e in generale gli agiti mossi da egocentrica spavalderia e pretenziosità. Il reato sembra non avere una 
costruzione, nasce senza una reale motivazione né una progettazione e in genere non viene interrotto da un ripensamento, ma da cause esterne, quali l'essere scoperti in flagrante o avere la peggio. Come detto, spesso la gravità e l'antigiuridicità delle azioni commesse si perde nel 'contenitore gruppo', così come la successiva riflessione sull'azione compiuta [47, 48, 49, 50, 51, 52].

La lettura dell'attuale delittuosità minorile non può prescindere da una analisi dello scenario storico-culturale entro il quale questi giovani faticosamente si muovono e crescono. La società contemporanea appare infragilita dalla perdita di significatività ed incisività degli strumenti di controllo sociale, a partire dalla famiglia, che attraversa una crisi senza precedenti, tangibile e preoccupante, foriera di una confusività valoriale all'interno della quale il giovane contemporaneamente si perde e si impone [14, 53, 54, 55, 56].

I genitori arrancano sempre più nell'esercizio del loro ruolo pedagogico: spesso - nella difficoltà di gestire figli pretenziosi e precocemente autonomizzati - ricorrono ad un approccio educativo oscillante tra una ipernormatività sterile e retorica o - di contro - una permissività in cui si pongono in simmetria con il figlio, nella purtroppo frequente posizione del "per te sono come un amico". La generazione dei nativi digitali non vive e non conosce più l'impalcatura tradizionale della famiglia, sperimentando invece spesso una struttura familiare allargata. Tale assetto si caratterizza talora per convivenze brevi e molteplici dei genitori con nuovi partner, situazioni che condizionano i ragazzi a vivere in promiscuità con adulti di passaggio, per loro estranei.

La sfiducia negli adulti e la conseguente ribellione nei confronti dei contenuti da loro (non) trasmessi, si legge attualmente persino nei nuovi scenari che si profilano all'interno della delinquenza organizzata, alla cui inflessibile regolamentazione sfuggono le azioni dei più giovani. Essi, infatti, sempre più di frequente rifiutano di sottostare alla rigida organizzazione gerarchica, di rispettarne i ruoli, e divengono ‘schegge impazzite' che agiscono in autonomia, soggetti particolarmente pericolosi proprio in quanto svincolati dagli adulti i quali - seppur criminali, spesso molto pericolosi - agiscono con raziocinio e pianificazione [41].

Per il gruppo sociale degli adulti, tuttavia, non è facile effettuare un'analisi che ne metta in discussione l'assetto, e la tendenza è quella di auto-assolversi, di legittimarsi ad effettuare una troppo facile - e deresponsabilizzante per la società stessa - lettura del giovane deviante e delle sue azioni antisociali come il frutto e l'espressione di un immanente ed inevitabile male contemporaneo, di cui solo il delinquente, o al limite la sua appartenenza familiare e sottoculturale, appaiono colpevoli. Il 'crollo dei valori', il malfunzionamento degli strumenti di controllo sociale, appaiono, al contrario, come l'espressione del malfunzionamento del gruppo stesso, che si disorienta quando le letture 
criminogenetiche tradizionali non offrono risposte nelle situazioni in cui il crimine sia svincolato da condizioni di marginalità sociale [4S].

È evidente, pertanto, come la complessità e la fragilità alla base di molte condotte criminose commesse da adolescenti richiedano oggi di individuare chiavi di lettura attualizzate, al fine di intervenire sulle disfunzionalità attraverso adeguati strumenti di general e specialprevenzione.

Le ferite dell'adolescente possono esprimersi in molte e diverse forme. Le dipendenze - da sostanze psicotrope, da cibo, da videogiochi, da utilizzo dei social network - possono essere considerate strategie per garantire la vicinanza tra sé e un oggetto di desiderio controllabile e disponibile, che non può essere perduto [57]. Il meccanismo può arrivare ad assumere risvolti preoccupanti e a produrre sintomi e condizioni pericolose per il ragazzo, come accade nei casi dei disturbi dell'alimentazione, nelle tossico o alcoldipendenze, nel rapporto patologico con i devices o con l'utilizzo dei social che si accompagna ad una forma esasperata di ritiro sociale. Si pensi alla cosiddetta 'Hikikomori Syndrome', condizione osservata per la prima volta qualche decennio fa in adolescenti giapponesi ed ora diffusa in tutto il mondo, che descrive un modus vivendi del ragazzo caratterizzato da completo e prolungato ritiro sociale, permanenza in ambiente domestico - nella propria comfort zone - dedizione esasperata e ripetitiva all'uso dei devices elettronici, perdita di interesse nei confronti di amicizie e in generale della socialità al di fuori dei contatti digitali $[46,47,58,59,60]$.

\section{Considerazioni conclusive}

La testimonianza del minore presenta aspetti di estrema delicatezza e complessità, che traggono origine dalla vulnerabilità connaturata ad un soggetto la cui struttura di personalità, proprio perché ancora in fase di costruzione e consolidamento, è suscettibile di venire influenzata negativamente da esperienze complesse, come le dinamiche che derivano dalla vicenda penale. Parallelamente, il compito dell'esperto in questo campo è gravato da aspetti di particolare difficoltà, che attengono a numerosi profili di tale intervento professionale, sia di carattere metodologico che clinico ma anche, e forse soprattutto, deontologico.

In questa cornice si segnala l'importanza del contributo della ricerca e della riflessione scientifica criminologica, segnatamente nella particolare situazione che si crea allorché il minore - sovente all'interno di un reato di gruppo, ma pure con la compartecipazione o l'influenzamento di una figura adulta, che può essere anche un familiare o un genitore, soprattutto nei contesti della 
criminalità organizzata - riveste il duplice ruolo di testimone e di autore di reato.

In questo quadro, inoltre, possono sempre più spesso interferire ulteriori fattori quali, in primis, gli importanti cambiamenti che interessano la cultura sociale nel nostro momento storico, cambiamenti che investono anche i modelli proposti ai membri del gruppo sociale e particolarmente rilevanti per i minori, ancora impegnati nel loro percorso evolutivo. Unitamente a ciò, le stimolazioni incalzanti che derivano dagli strumenti della comunicazione multimediale facilitano il contatto del minore con innumerevoli settori di sollecitazione che possono porre, soprattutto per una struttura di personalità ancora immatura, specifiche difficoltà nelle quali possono intrecciarsi i ruoli di testimone ed autore di reato, oltre che ovviamente di vittima.

La commissione del reato di gruppo, segnatamente, interessa differenti aree che influenzano l'agire dell'adolescente e che si possono riverberare anche sull'azione testimoniale, facilitando posizioni che talora si intrecciano fra loro, rispetto al reato: autore, testimone, persona informata sui fatti. Il legislatore fornisce la cornice tecnico-giuridica per il posizionamento di tali ruoli, i quali tuttavia pongono numerosi, ulteriori e delicati problemi, le cui composite sfaccettature richiedono una lettura più articolata che si avvalga anche del contributo della riflessione criminologica. Quest'ultima, in particolare, può fornire un contributo nella prospettiva della prevenzione, primaria (sul piano della cultura sociale e dell'accompagnamento educativo, familiare ed attraverso le strutture istituzionali e territoriali) e secondaria, attraverso la formazione dei professionisti e dei tecnici che intervengono in questo delicato settore, rivolto all'accompagnamento ed alla tutela del minore.

\section{Bibliografia}

[1] Mazzoni G. Si può credere a un testimone? La testimonianza e le trappole della memoria. Bologna: il Mulino; 2003.

[2] Pansini C. Le dichiarazioni del minore nel processo penale. Padova: Cedam; 2001.

[3] Fornari U. Trattato di Psichiatria forense. Milano: Utet; 2018.

[4] Capri P. La valutazione della capacità a testimoniare dei minori nei processi penali in materia di abuso sessuale, Rivista Italiana di Medicina legale 2011, VI: 1647-56.

[5] De Cataldo Neuburger L, a cura di. Testimoni e testimonianze deboli. Padova: Cedam; 2006.

[6] Goodman GS, Hirsman SE, Hepps D, Rudy L. Children's Memory for Stressful Events. Morril Palmor Quarterly 1991, 37: 109-47. 
[7] Grattagliano I, Carabellese F, Berlingerio I, Lisi A, Mongelli RG, Catanesi R. Ingresso nei ricordi. Una ricerca sulla suggestionabilità dei bambini. Rassegna Italiana di Criminologia 2010, 1: 145-59.

[8] Recchione S. L'ascolto del minore nel processo penale, Rivista Italiana di Medicina Legale 2011, VI: 1639-46.

[9] Mazzoni G, a cura di. La testimonianza nei casi di abuso sessuale sui minori. Milano: Giuffrè; 2000.

[10] Elischberger HB. The Effect of Prior Knowledge on Children's Memory and Suggestibility, Journal of Experimental Child Psychology 2005, 92: 247-75.

[11] De Cataldo Neuburger L, Gulotta G. La Carta di Noto e le linee guida deontologiche per lo psicologo giuridico. Milano: Giuffrè; 2004.

[12] Conti A. Le garanzie processuali del minore: un confronto tra l'ordinamento europeo e la disciplina italiana. Minorigiustizi. 2019, 2: 96-107.

[13] Sibilio D. Secondo la Corte europea lo Stato ha il dovere di proteggere i minori vulnerabili anche da se stessi. Rivista italiana di diritto e procedura penale 2018: 980-4.

[14] De Leo G. La giustizia dei minori: la delinquenza minorile e le sue istituzioni. Torino: Einaudi; 1981.

[15] Bargis M, a cura di. Procedura penale minorile. Torino: Giappichelli Editore; 2019.

[16] Moro AC. Manuale di diritto minorile. Zanichelli: Bologna; 2008.

[17] Cavazza C, Perduca R, Zandrini S. Adolescenti autori di reato: il processo psicosociale in ambito istituzionale. Milano: FrancoAngeli; 2019.

[18] Merzagora Betsos I. Ampiezza, andamento, tipologie della delinquenza minorile e giustizia riparativa. In: Picotti L, a cura di. La mediazione nel sistema penale minorile. Padova: Cedam; 1998.

[19] Marshall TF. Restorative justice. An overview. Bachinghamshire: Home office; 1999.

[20] Ellenberger F. Relations psychologiques entre le criminal et la victim. Revue Internationale de Criminologie et de Police Technique 1954, 8:103-6.

[21] Ceretti A, Di Ciò F, Mannozzi G. Giustizia riparativa e mediazione: esperienze e pratiche a confronto. In: Scaparro F, a cura di. Il coraggio di mediare: contesti, parodie e pratiche di risoluzioni alternative alle controversie. Milano: Guerini e Associati; 2001.

[22] Romano CA. Dal modello rieducativo a quello riparativo: spunti e applicazioni. Rassegna Italiana di Criminologia 2012, 2: 103-15.

[23] Salvatore A. La giustizia minorile nel '900. Milano: Unicopli; 2007.

[24] Barbero Avanzini B. Minori giustizia penale e intervento dei Servizi, Milano: FrancoAngeli; 2001.

[25] Lanza E. La sospensione del processo con messa alla prova dell'imputato minorenne. Giuffrè: Milano; 2003.

[26] Giostra G, a cura di. Il processo penale minorile, Milano: Giuffrè; 2016.

[27] Tribisonna F. L'ascolto del minore testimone o vittima di reato nel procedimento penale. Padova: Cedam; 2017.

[28] Gulotta G. Elementi di psicologia giuridica e di diritto psicologico. Milano. Giuffrè; 2000. 
[29] Pazè P. L'ascolto del bambino nell'adozione nazionale, Minorigiustizia 2001, 1: 50-65.

[30] Cesari C. "Il minore informato sui fatti" nella legge n. 172/2012. Rivista Italiana di diritto e procedura penale 2013, 1: 157-93.

[31] De Cataldo Neuburger L. La testimonianza del minore. Cedam, Padova. 2005.

[32] Famiglietti A. La testimonianza del minore di sedici anni in incidente probatorio ed il raggiungimento della maggiore età. Rivista Italiana di Diritto e Procedura Penale 2004, 1: 289-98.

[33] Giostra G. La testimonianza del minore: tutela del dichiarante e tutela della verità. Rivista Italiana di Diritto e Procedura Penal, 2005, 3: 1019-1028

[34] Siracusano F. Indagini difensive e "persona informata" di minore età. In Cesari C, a cura di. Il minore fonte di prova nel processo penale, Giuffrè, Milano; 2008.

[35] Ardita S. La prevedibilità ex art. 512 c.p.p. dei fatti impeditivi della ripetizione della testimonianza in dibattimento. Il caso del minore affetto da grave forma di stress, Cassazione Penale 2002, 2: 614.

[36] Cassibba F. La tutela dei testimoni “vulnerabili”, In: Matza O, Viganò F, a cura di. Il pacchetto sicurezza 2009, Giappichelli, Torino; 2009.

[37] Daniele M. Regole di esclusione e regole di valutazione della prova. Giappichelli. Torino; 2009.

[38] Grevi V. Le "dichiarazioni rese dal coimputato" nel nuovo codice di procedura penale, Rivista Italiana di Diritto e Procedura Penale 2009, 3: 1175-6.

[39] Ellenberg H. F. Relations psycologiques entre le criminal et la victim. Revue international de criminology et de police technique 1954, 8: 103-106.

[40] Cagli S. Condotta della vittima e analisi del reato. Rivista Italiana di Diritto e Procedura penale 2000, 3: 1148-58.

[41] Schermi M. L'educazione criminale: crescere in contesti mafiosi. Rassegna italiana di criminologia. 2013, 4: 256-263.

[42] Belotti V. Maurizio R. Moro A. C. Minori stranieri in carcere. Milano, Guerini, 2006.

[43] Mastropasqua I. I ragazzi stranieri nel sistema della giustizia penale italiana. Rassegna italiana di criminologia 2013, 4: 243-55.

[44] Tomai G. I minori stranieri non accompagnati fra tutela ed accoglienza. Minorigiustizia 2019, 1: 42-50.

[45] Dell'Amico F. Essere un minorenne autore di reato in Africa. La realtà del Burkina Faso e del Malawi. Minorigiustizia, 2008, 4, 279-90.

[46] Gualco B. Fossa G. Malagoli S. Orlandi E. Salvadori L. Rensi R. Presenza dei genitori e vittimizzazione dei giovani in Italia. Risultati dell'international selfreport delinquency Studi-3. Rassegna italiana di criminologia 2019, 1: 30-38.

[47] Sabatello U. Giacolini T. Thomas F. Condotte aggressive e antisociali nell'infanzia e nell'adolescenza: alcune riflessioni cliniche e psicopatologiche. Rassegna italiana di criminologia 2018, 3: 231-246.

[48] Camerini G. B. Volterra V. Criteri di valutazione delle capacità genitoriali e strumenti operativi. Rassegna italiana di criminologia 2007, 3: 135-151.

[49] Amir M. Patterns in forcible rapes. Chicago. University of Chigago. Press; 1971.

[50] Zara G. Veggi S. I minorenni sessualmente abusanti tra solo-offending e cooffending. Uno studio operativo. Rassegna italiana di criminologia 2018, 4: 262-274. 
[51] Gatti U. Rocca G. Verde A. Devianza giovanile e giustizia minorile, fra tradizione e cambiamento. Rassegna italiana di criminologia, 2013, 4: 234-235.

[52] Rusconi M. Il trattamento degli adolescenti abusanti, tra responsabilità etica e giudizio morale, Minorigiustizia 2009: 3: 115-123.

[53] Maggiolini A. Riva E. Adolescenti trasgressivi. Le azioni devianti e le risposte degli adulti, Milano, FrancoAngeli; 2003.

[54] Camerini G. B, Volterra V. Criteri di valutazione delle capacità genitoriali e strumenti operativi. Rassegna italiana di criminologia 2007; 3: 135-152.

[55] Susanna Pietralunga, Claudia Salvioli, Ivan Galliani. Reati violenti commessi da minorenni. La vulnerabilità biologica, psichica e sociale del minore”. Rassegna italiana di criminologia. 2010: 2: 237-253.

[56] Bandini T, Gatti U. Dinamica familiare e delinquenza giovanile. Milano: Giuffrè Editore; 1972. p. 35-84: 191-201.

[57] Di Nunno N. Lamboni V. Boninfante B. Pino J. Greco O. Il consumo di sostanze psicoattive tra gli adolescenti italiani. Rassegna italiana di criminologia. 2015, 3: 205-218.

[58] Toniatti C. Maiocchi L. M. Genitori e figli nell'era di internet: comunicazione reale e comunicazione virtuale, Minori giustizia, 2009: 4: 190-203.

[59] Pozza A. Coluccia A. Gaetano M. Gusinu R. Ferretti F. La sindrome di Hikikomori: prevalenza nella popolazione generale e psichiatrica. Una sistematic review con meta-analisi. Rassegna italiana di criminologia, 2019: 1, 6-17.

[60] Caffo A, Catanesi R, Greco R, Grattagliano I, Toma E, Taurino A. Il ruolo di internet e degli strumenti informatici di comunicazione sulla qualità delle relazioni tra preadolescenti. Una ricerca pilota. Rassegna italiana di criminologia, 2017, 1: 67-75.

\section{Sitografia}

[1S] Baiguera Alieri A. Analisi comparata delle delinquenze giovanili in Italia ed in Svizzera. Diritto.it 15 maggio 2018.

[2S] Bonaccorso W. Il minore autore, vittima o testimone di un reato, https://www. diritto.it/author/walter-bonaccorso/ 6 aprile 2018.

[3S] Lanotte A., Di Cosimo L. Accertamento della personalità del minore, www. aipgitalia.org, 2002.

[4S] Maggia C. La sicurezza tra paura e diritti. Intervento tenuto a Parole di Giustizia 2016, Sessione Sicurezza, intervento giudiziario, diritti, (I) Lo Stato e il nemico: reprimere; (II) Lo Stato e il nemico: riconciliare, La Spezia 15/16 aprile 2016, seminario annuale dell'Associazione studi giuridici Giuseppe Borré.

\section{Per corrispondenza:}

Email: susanna.pietralunga@unimore.it 